\title{
Prozessorientiertes Fallmanagement in der traumatologischen Rehabilitation*
}

\author{
Gerade in den letzten Jahren ist festzustellen, dass Patienten zunehmend \\ früher aus den Akutspitälern Rehabilitationskliniken zugewiesen werden, wes- \\ halb auch die «Weiterbehandlung» dieser betroffenen Menschen in die um- \\ fassenden Behandlungskonzepte der Rehabilitation integriert werden muss. \\ Inhaltlich weichen Behandlungsziele, -strategien und -inhalte der Rehabilita- \\ tionsmedizin aber deutlich von der Akutmedizin ab. Deshalb lässt sich die \\ Rehabilitation nicht auf die Fortführung der Akutmedizin im Sinne einer «Wei- \\ terbehandlung» oder gar einer einfacheren «Nachbehandlung» reduzieren.
}

Hans Peter Gmünder ${ }^{a}$, Sönke Johannes ${ }^{b}$

a Dr. med., Chefarzt und Medizinischer Direktor

b Professor Dr. med., Stv. Medizinischer Direktor und Leitender Arzt Neurorehabilitation

Eine ausführliche Version dieses Artikels wird in «Medizinische Mitteilungen 77», herausgegeben von der Suva, Redaktionsleitung Chefarzt Suva, voraussichtlich im Mai/Juni 2006 erscheinen.

Korrespondenz:

Rehaklinik Bellikon

CH-5454 Bellikon

hanspeter.gmuender@

rehabellikon.ch

soenke.johannes@rehabellikon.ch
In der Rehabilitation versuchen wir grundsätzlich, die verlorengegangenen Funktionen und Fähigkeiten unserer Patientinnen und Patienten wiederherzustellen. Hierdurch sollen die Patienten befähigt werden, ihre verschiedenen Rollen in der Gesellschaft bestmöglich und eigenständig ausüben zu können. In diesem Sinne ist es erforderlich, Behandlungsziele zu berücksichtigen, die der reinen Funktion übergeordnet sind. Es handelt sich um Partizipations- bzw. Teilhabeziele. Sollte die Wiederherstellung von Funktionen und Fähigkeiten aber aufgrund der Schwere oder Komplexität der Schädigung nicht oder nur teilweise möglich sein, müssen die Ziele der Rehabilitation darauf ausgerichtet werden, den betroffenen Menschen ein Leben mit Folgeschäden zu ermöglichen. Eine bestmögliche Integration und damit Teilhabe an beruflichen und soziokulturellen Lebenssituationen gelingt in diesen Fällen nur durch den Einsatz verschiedener spezialisierter Berufsgruppen auf der Basis von umfassenden biopsychosozialen Behandlungsstrategien (= Berücksichtigung der verschiedenen Dimensionen von Behinderung und gesundheitlicher Integrität als Grundlage eines mehrdimensional orientierten Behandlungskonzeptes).

Bei dieser Betrachtung von Rehabilitation ist es wichtig, die einfachere - und in der Regel meistens nur eindimensional auf die medizinischen Aspekte ausgerichtete - Nachbehandlung abzugrenzen. Hierfür sind in der Regel ambulante Strukturen ausreichend, sofern die Selbständigkeit der betroffenen Patienten nicht wesentlich beeinträchtigt ist.

\section{ICF in der Rehabilitation}

Die Internationale Klassifikation der Funktionsfähigkeit, Behinderung und Gesundheit (ICF, Originaltitel: International Classification of Functioning, Disability and Health) ist ein Klassifikationssystem der WHO im Rahmen «einer Familie von Klassifikationssystemen für die Anwendung auf verschiedene Aspekte der Gesundheit». Die ICF ermöglicht es, die unterschiedlichen Dimensionen von gesundheitlicher Integrität und Behinderung in einer einheitlichen Sprache systematisch zu erfassen. Somit kann die ICF nicht nur bei Menschen mit Behinderung angewendet werden. Sie gilt für alle Menschen und kann die Betrachtungsebene der ICD-10 (International Statistical Classification of Diseases and Related Health Problems, Tenth Revision) ideal ergänzen («Zwei Personen mit derselben Krankheit können ein unterschiedliches Niveau der Funktionsfähigkeit aufweisen, und zwei Personen mit gleichem Niveau der Funktionsfähigkeit haben nicht notwendigerweise das gleiche Gesundheitsproblem»). Aufgrund ihrer mehrdimensionalen Betrachtungsweise ist das Gedankengut der ICF für die Problemanalyse, Triagierung und Prozesssteuerung in der Rehabilitationsmedizin sehr geeignet.

Folgende Komponenten liegen der ICF zugrunde:

- Funktionen und Strukturen des menschlichen Organismus;

- verschiedenste Aktivitäten einer Person;

- Teilhabe an Lebensbereichen (interpersonelle Beziehungen, Erziehung/Bildung, Arbeit und Beschäftigung, soziales und staatsbürgerliches Leben usw.). 
Diese drei Komponenten werden in Beziehung gesetzt zu den sogenannten Kontextfaktoren, die sich in Umweltfaktoren und Personbezogene Faktoren (wegen grosser soziokultureller Unterschiede nicht klassifiziert) unterteilen und den Lebenshintergrund eines Menschen darstellen.

\section{Rehabilitationsprozess}

Am Beginn des Rehabilitationsprozesses steht die Problemanalyse am Patienten (Abb. 1), wobei neben einer diagnosebezogenen Betrachtung der systematische Einbezug der ICF äusserst wichtig ist.

Tabelle 1

ICF-basierte Zielkategorien.

I Akutrehabilitation und Lebensqualität

Medizinische Abklärung und Behandlung

Betreutes Wohnen

Wohnen zu Hause

Teilhabe am soziokulturellen Leben

Arbeit in geschütztem Rahmen

II Berufliche Integration

Berufliche/schulische Reintegration in späterer Rehaphase

Umschulung

Stellensuche

Neue Tätigkeit im bisherigen Betrieb

Angestammte Tätigkeit im bisherigen Betrieb

III Medizinische Expertisen

Zumutbarkeitsbeurteilung/Standortbestimmung/

Vorbereitung Fallabschluss

Gutachten
Die Problemanalyse muss somit neben einer herkömmlichen Anamnese und Untersuchung durch den Arzt systematisch die wesentlichen Aspekte von Funktion, Leistungsfähigkeit, Teilhabe und Kontextfaktoren gemäss ICF einschliessen. Dies gelingt durch den Einbezug weiterer Fachgruppen (z. B. Physiotherapie, Ergotherapie, Neuropsychologie, Logopädie, Pflege) in ein standardisiertes Vorgehen mit definierten Assessmentsystemen. Hierbei handelt es sich um semistrukturierte Interviews, praktisch-klinische Observationen und Untersuchungen sowie um die Anwendung von ausgewählten Testverfahren (Assessments). So werden beispielsweise in unserer Neurorehabilitation bei der Beurteilung der ICF-Kategorie «Mobilität» ergänzend zu den ärztlichen und therapeutischen Befunden gezielt weitere Testverfahren und Fragebogen (Chedoke McMaster Test, 3-Minuten-Gehtest, Handkoordinationstest usw.) eingesetzt. Solche zusätzlichen Abklärungen sind aufgrund einer initialen Patientengruppierung, die auf unserem System «Zielkategorien» (s.u.) beruht, vordefiniert.

In einem zweiten Schritt sollte zusammen mit dem Patienten das langfristige Teilhabeziel verbindlich festgelegt werden. Hierfür ist es sinnvoll, wenn auf einen Katalog von definierten Teilhabezielen («Zielkategorien») zurückgegriffen werden kann. An der Rehaklinik Bellikon arbeiten wir in unseren drei Kerngeschäftsbereichen Traumatologische Akutrehabilitation, Berufliche Integration und Medizinische Expertisen mit 12 Zielkategorien (Tab. 1).

Eine Zuordnung der Patienten zu definierten Teilhabezielen ist nicht nur im Hinblick auf einen systematischen und - soweit sinnvoll standardisierten Rehabilitationsprozess notwen-

Abbildung 1

Rehabilitationsprozess schematisch.

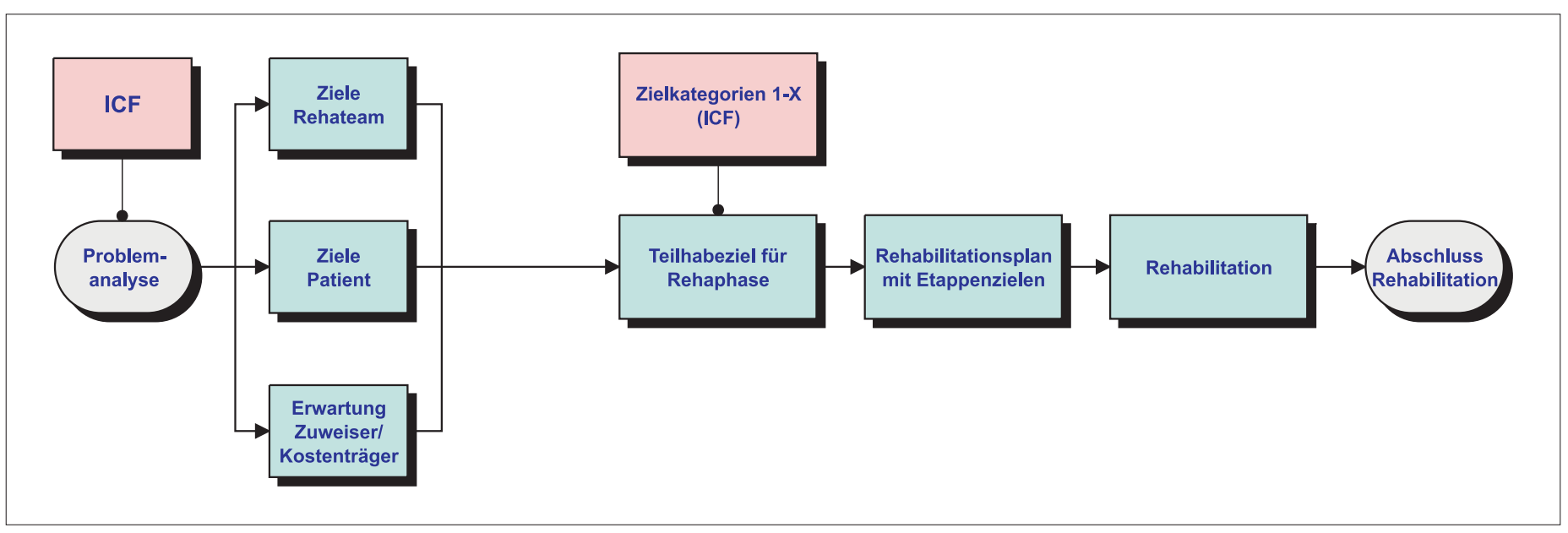


** Jeder dieser Teilprozesse ist durch etwa 20 Aktivitäten und Messgrössen definiert. dig, sondern auch im Hinblick auf verschiedenste Ergebnisanalysen (medizinischer Outcome, Kosten- und Ertragsanalysen, Patientenzufriedenheit).

In diesen auf die Teilhabe bezogenen Zielkategorien muss präzise festgelegt sein, welche Kriterien bezüglich der anderen ICF-Komponenten (Körperfunktionen und -strukturen, Aktivitäten, Umweltfaktoren und Personbezogene Faktoren) bis zum Abschluss der aktuellen Rehabilitationsphase erfüllt sein müssen (Tab. 2).

Abbildung 2

Behandlungspfad bestehend aus verschiedenen Komponenten (Teilprozessen**) am Beispiel Amputation untere Extremität (blau), Zielkategorie «Umschulung» (rot).

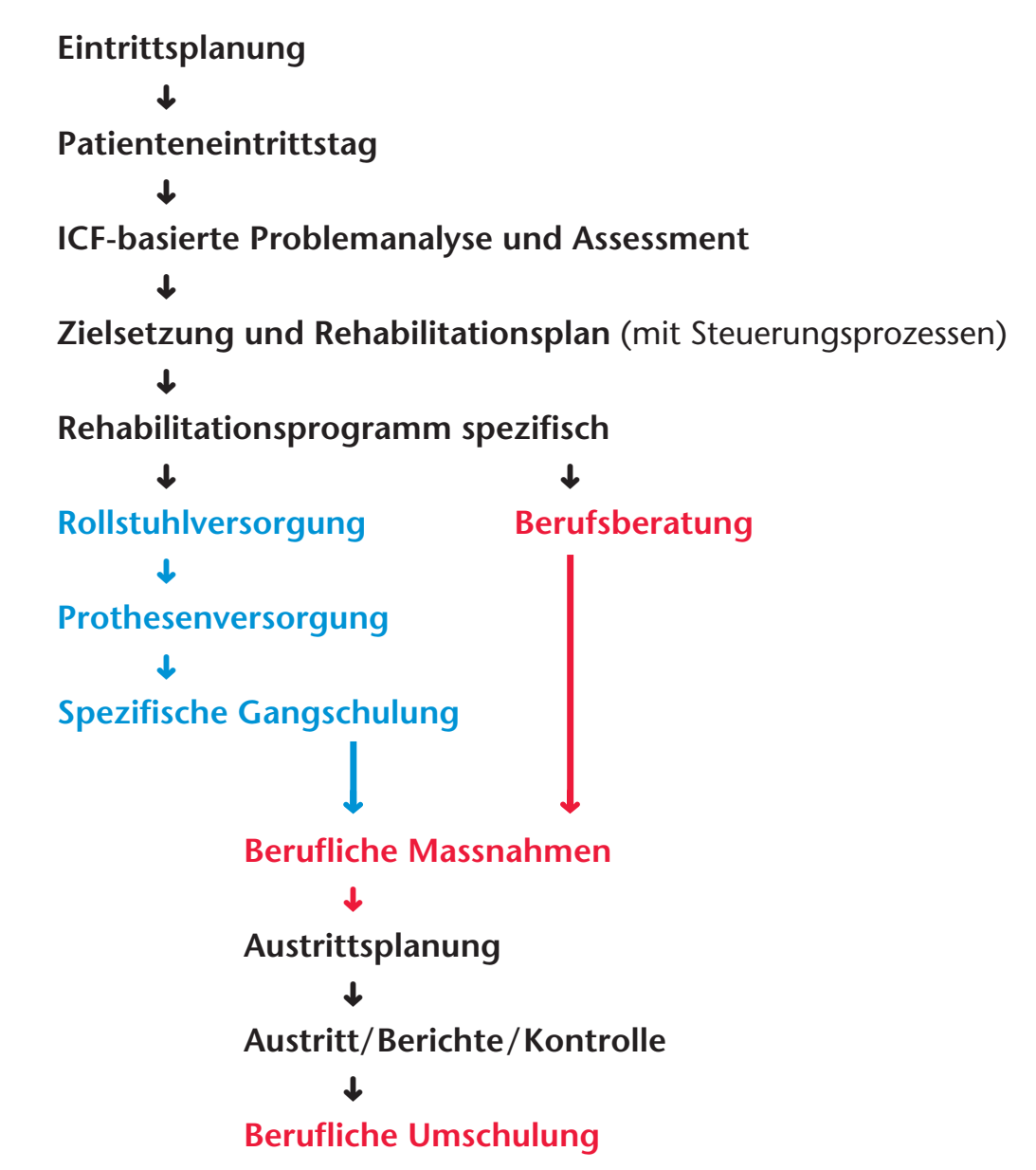

Tabelle 2

Hauptkriterien am Beispiel der Zielkategorie «Umschulung».

Körperfunktionen/-strukturen Diagnose für neue Tätigkeit nicht limitierend
Aktivitäten

Belastbarkeit für neue Tätigkeit mindestens 4 Std. an einem halben Tag
Zur Erstellung eines detaillierten Rehabilitationsplanes ist es notwendig, Schlüsselziele (möglichst standardisiert) und kurzfristigere Etappenziele (oft Wochenziele) in den einzelnen Berufsgruppen zu formulieren, wobei diese einen klaren Bezug zu der festgelegten Zielkategorie und geplanten Aufenthaltsdauer haben müssen.

\section{Behandlungspfade in der Rehabilitation}

Medizinische Leitlinien bilden eine wichtige Grundlage für die Erstellung von Diagnose- und Behandlungspfaden, welche die spezifischen Gegebenheiten einer Klinik oder Institution berücksichtigen. Auf diese Weise ist der «Pfad» eines Patienten mit einer bestimmten Diagnose im Hinblick auf Abklärung und Behandlung präzise und verbindlich festgelegt. In der Rehabilitation ist ein solches Vorgehen in bezug auf die medizinischen Diagnosen nur bedingt sinnvoll, da sich die Behandlungsziele und -massnahmen nur teilweise aus solchen Diagnosegruppierungen ableiten lassen. Entscheidend ist in der Rehabilitation die Problemanalyse unter Berücksichtigung der verschiedenen Dimensio-

Die Rehaklinik Bellikon der Suva ist die Spezialklinik für Traumatologische Akutrehabilitation, Berufliche Integration und Medizinische Expertisen. Sie behandelt Allgemein-, Halbprivat- und Privatversicherte Suva- und Nicht-Suva-Patienten im 24-Std.Betrieb. Dank ihrer Überwachungsstation, den Spezialzimmern für Brandverletzte und Patienten ohne Arme und Hände bietet sie eine umfassende Rehabilitation auf einem Toplevel. Sie offeriert 206 Betten in 1- und 2-Bett-Zimmern (in Ausnahmefällen 3-BettZimmer) in einem modernen Ambiente mit zeitgemässer Gastronomie und Hotellerie.

Die Rehaklinik Bellikon wird mit einem Referat, einem Podiumsgespräch und einem Informationsstand an den Luzerner Trendtagen Gesundheit am 23. und 24. März 2006 im KKL Luzern (siehe auch www.trendtagegesundheit.ch) vertreten sein.

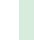

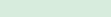
Umwelt-/Personbezogene Faktoren
Patient hat Anspruch auf IV-Massnahmen (selten anderer Versicherungs-
träger); ausreichende persönliche Ressourcen (Alter, Bildung, Sprachkennt- nisse, Ausbildung, Beruf usw.)


Abbildung 3

Adaptiertes Klinikinformationssystem zur Prozesssteuerung.

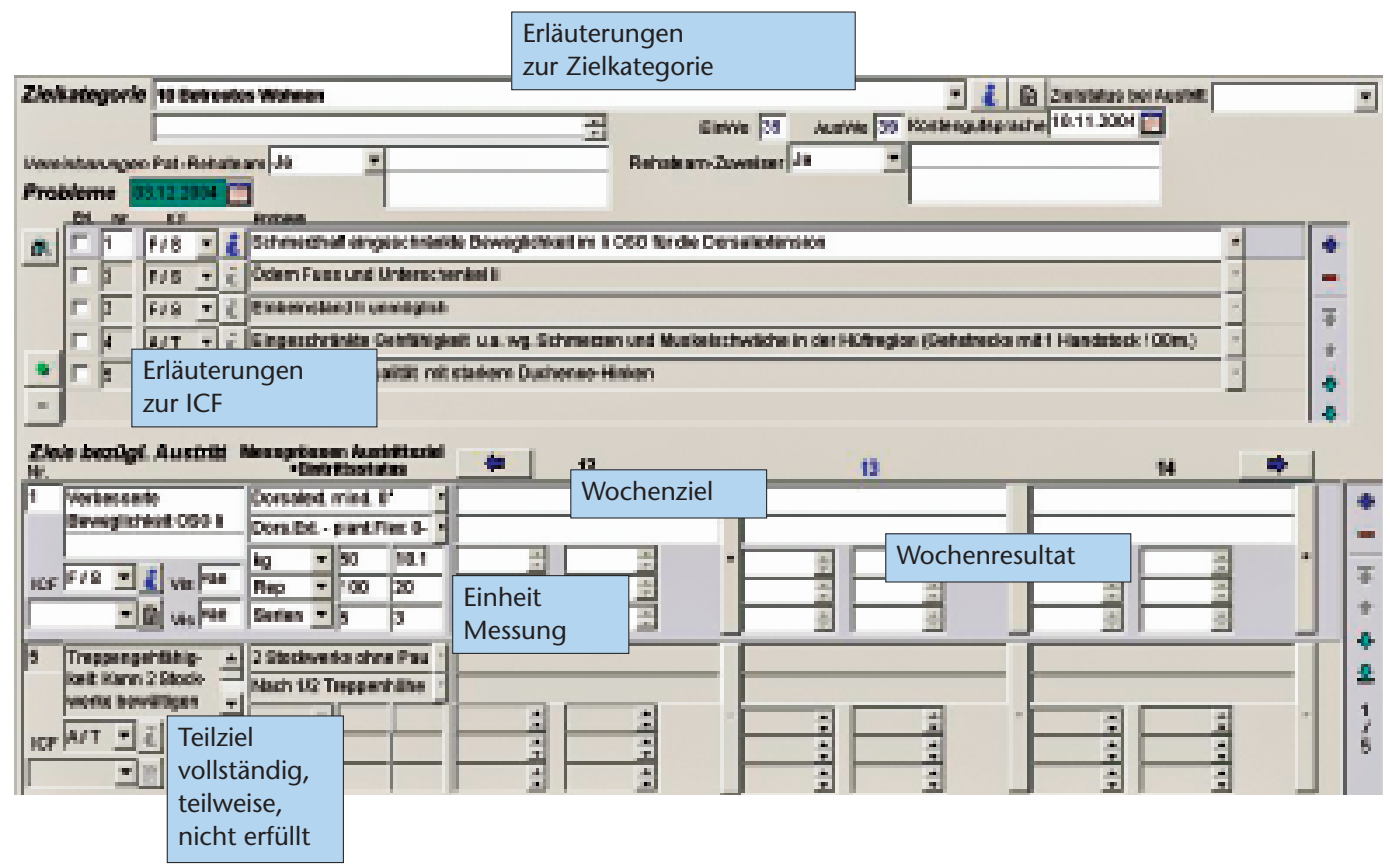

nen von gesundheitlicher Integrität und Behinderung eines Patienten einschliesslich der Kontextfaktoren (s.o.). Neben einem allgemeinen Behandlungspfad kann durch die Verknüpfung mit medizinischen Diagnosen und ICF-basierten Teilhabezielen («Zielkategorien») der Behandlungspfad präzisiert werden und vordefinierten, ressourcenorientierten Behandlungsprogrammen zugeordnet werden (Abb. 2).

Ein solches systematisches Vorgehen ermöglicht die Auswahl eines individuell angepassten Rehabilitationsprogrammes, das auf standardisierten Vorgaben basiert. Darüber hinaus bleibt auch dann die Möglichkeit für eine weitere individuelle Ausgestaltung des Rehabilitationsprozesses, wobei diese wiederum nicht zufällig erfolgen darf, sondern nach einem festgelegten Vorgehen, das in den gesamten Rehabilitationsprozess integriert ist. In der Rehaklinik Bellikon geschieht dies über einen definierten Zielsetzungsprozess, der Rückkopplungs- und Steuerungsmechanismen sowie Ergebnis- und Prozessmessgrössen zur Qualitätskontrolle und Qualitätsverbesserung enthält. Für die praktische Umsetzung wurde das elektronische Klinikinformationssystem entsprechend angepasst (Abb. 3).

\section{Literatur}

- Andresen EM. Criteria for assessing the tools of disability outcomes research. Arch Phys Med Rehabil 2000;81(Suppl 2):15-20.

- Barnes MP, Ward AB. Textbook of Rehabilitation Medicine. New York: Oxford University Press; 2000 .

- Braddom RL. Physical Medicine and Rehabilitation. $2^{\text {nd }}$ edition. Philadelphia, London, New York: Saunders; 2000.

- Ewert T, Cieza A, Stucki G. Die ICF in der Rehabilitation. Phys Med Rehab Kuror 2002;12:157-62.

- Gmünder HP. Rehabilitation. In: Rüter A, Trentz O, Wagner M. Unfallchirurgie. München: Urban \& Fischer; 2004. S. 443-50.

- Haaf HG. Gesundheitsökonomische Analyse der Vergütung mit Fallpauschalen in der medizinischen Rehabilitation. Rehabilitation 2002;41:14-30.

- Jette AM, et al. Beyond function: predicting participation in a rehabilitation cohort. Arch Phys Med Rehabil 2005;86: 2087-94

- Kaplan RM. Quality of life: An outcomes perspective. Arch Phys Med Rehabil 83, Suppl 2: 44-60; 2002

- Knüsel O. ALVR-Grundlagen der Rehabilitation. Schweiz Ärztezeitung 2002;83(37):1923-4.

- Köhler F. Auswirkungen des DRG-Systems auf Anschluss- und Rehabilitationsbehandlung in Sydney, New South Wales, Australien. Rehabilitation 2002;41:10-3 
- Müller K, Schneider W. Autonomie und soziale Integration. Geriatrie Praxis 2003;3:28-30; Arch Phys Med Rehabil 2002;83(Suppl 2):44-60.

- Rentsch HP, Bucher PO. ICF in der Rehabilitation. Idstein: Schulz-Kirchner; 2005.

- Rochell B, Roeder N. DRGs als Grundlage der künftigen Krankenhausfinanzierung - Stand der Umsetzung und Einfluss auf die Rehabilitation. Rehabilitation 2002;41:1-9.

- Rushton PW, Miller WC. Goal attainment scaling in the rehabilitation of patients with lowerextremity amputations: a pilot study. Arch Phys Med Rehabil 2002;83:771-5

- Schuntermann MF. Die Bedeutung der Internationalen Klassifikation der Funktionsfähigkeit, Behinderung und Gesundheit (ICF) für die Rehabilitation. Med Sach 2003;99(3):94-7.

- Schut HA, Stam HJ. Goals in rehabilitation teamwork: Disabil Rehabil 1994;16(4):223-6.
- Sivaraman Nair KP, Wade DT. Satisfaction of members of interdisciplinary rehabilitation teams with goal planning meetings. Arch Phys Med Rehabil 2003;84(Suppl 2):1710-3.

- Stoll T, et al. Sind stationäre Rehabilitation oder ambulante Physiotherapie bei Patienten mit muskuloskeletalen Problemen wirkungsvoll und landen Patienten auf dem richtigen Behandlungspfad? Physiotherapie 2004;4:17-21.

- Stucki G, Sigl T. Assessment of the impact of disease on the individual. Best Pract Res Clin Rheumatol 2003;17(3):451-73.

- Whiteneck GG, et al. Quantifying environmental factors: a measure of physical, attitudinal, service, productivity, and policy barriers. Arch Phys Med Rehabil 2004;85:1324-35.

- World Health Organisation. International Classification of Functioning, Disability and Health (ICF) Geneva; 2001. Entwurf der deutschsprachigen Fassung Mai 2002 (noch nicht publiziert).

\title{
L UZERNER \\ T T R N D TA GE \\ a E S UN D H E T \\ 2. Luzerner Trendtage \\ Gesundheit \\ Do/Fr, 23./24. März 2006, \\ KKL Luzern
}

\section{Herausforderung hochspezialisierte Medizin}

\author{
Machbarkeit - Finanzierbarkeit - Ethik
}

\section{Tagungsprogramm}

Die 2. Luzerner Trendtage Gesundheit widmen sich der hochspezialisierten Medizin. Das Thema ist heute eine der grössten Herausforderungen im Gesundheitswesen. Gerade in der Spitzenmedizin droht sich die Schere zwischen Machbarkeit und Finanzierbarkeit immer weiter zu öffnen. Da ist es dringend notwendig, die absehbaren Entwicklungen interdisziplinär und offen zu diskutieren. Der Kongress will dazu einen Beitrag leisten. Am Beispiel der Rehabilitation und der Onkologie soll zudem aufgezeigt werden, welche Auswirkungen die Spitzenmedizin auf andere Disziplinen hat und dass auch in weniger spektakulären Fachbereichen Spitzenleistungen erbracht werden.

\section{Veranstalter}

Träger der Veranstaltung ist das im Frühjahr 2004 gegründete Forum Gesundheit Luzern. Es steht unter dem Vorsitz des Luzerner Regierungsrats Dr. Markus Dürr. Der Verein ist bestrebt, die Luzerner Trendtage Gesundheit alljährlich durchzuführen. Er steht all jenen interessierten Kreisen zur Mitgliedschaft offen, welche die Ziele des Vereins unterstützen.

Vereinsmitglieder erhalten einen reduzierten Tagungstarif und sind zum Rahmenprogramm mit Nachtessen am Donnerstag abend, 23. März 2006 im Planetarium / Verkehrshaus der Schweiz eingeladen.

Credit-Points: SGGG, SGMO, SGPG, SGPMR, SRO.

\section{Information und Anmeldung}

Forum Gesundheit Luzern, Horwerstrasse 87, 6005 Luzern, Tel. 041318 37 97, Fax 04131837 10, E-Mail: info@trendtage-gesundheit.ch, Internet: www.trendtage-gesundheit.ch

Anmeldeschluss ist der 20. März 2006 